\title{
METATAXONOMIC ANALYSIS OF THE BACTERIAL DIVERSITY IN TABLE OLIVE DRESSING COMPONENTS
}

\author{
Antonio Benítez-Cabello ${ }^{\mathrm{a},}$, Verónica Romero-Gil ${ }^{\mathrm{b}}$, Eduardo Medina ${ }^{\mathrm{a}}$, Borja Sánchez ${ }^{\mathrm{c}}$, \\ Beatriz Calero-Delgado ${ }^{\mathrm{a}}$, Joaquín Bautista-Gallego ${ }^{\mathrm{a}}$, Rufino Jiménez-Díaz ${ }^{\mathrm{a}}$ \& Francisco \\ Noé Arroyo-López ${ }^{\mathrm{a}}$
}

${ }^{\text {a }}$ Food Biotechnology Department, Instituto de la Grasa (CSIC), Ctra. Utrera km 1, Building 46, 41013, Seville, Spain.

${ }^{\mathrm{b}}$ Oleica Technological \& Safety (TAFIQS in FOODs, S.L.), C/ Caña s/n, 29569Pizarra, Málaga, Spain.

c Department of Microbiology and Biochemistry of Dairy Products, Instituto de Productos Lácteos de Asturias (CSIC), Paseo Río Linares s/n, 33300-Villaviciosa, Principado de Asturias, Spain.

Running title: Bacterial diversity in olive dressings

*Corresponding author: Antonio Benítez Cabello. E-mail address: abenitez@ig.csic.es 


\section{Abstract}

2 In this work, we have used a metataxonomic analysis to study the bacterial biodiversity

3 in three types of commercial dressing and ingredients habitually used in table olive

4 packaging. The bioinformatics analysis showed that salt samples (marine and spring)

5 had the highest biodiversity indexes, followed by the aromatic herbs (thyme, oregano,

6 and fennel) and finally by fermented dressing samples (garlic, pepper, and pepper

7 paste). In salt samples, a significant high presence of Cytophagia, Sphingobacteria,

8 Deltaproteobacteria and Halobacteria classes was observed. On the contrary, the

9 presence of Bacilli class in fermented dressing samples was significantly higher

10 compared to the other samples. Finally, the abundance of Gammaproteobacteria was

11 statistically higher in the samples of herbs. At genus or family taxonomic level,

12 Pseudomonas and diverse non-assigned genus of the Enterobacteriaceae family

13 predominated in many samples of herbs. In fermented dressing samples, diverse genus

14 belonging to the Enterobacteriaceae family appeared in a high relative abundance in

15 pepper paste and garlic, and presence of Vibrio sp. was detected in pepper. However,

16 Lactobacillus, Weisella, and Pediococcus were the predominant genera found in many

17 of these fermented samples. Salinibacter was the most representative genus in both types of salt samples. Multivariate analysis (PCA) of the metataxonomic data sets discriminated well the samples belonging to the three different groups, showing a high proportion of sequences shared by samples-group. This survey reports for the first time

21 the presence of new bacterial species not previously related with table olive processing, as well as the presence of others which could compromise the stability of olive packaging.

24 Keywords: Table olives; Packaging; Metabarcoding; Seasoning ingredients; Salt 


\section{INTRODUCTION}

The olive tree is an iconic species in the Mediterranean cultural history and diet.

Its multiple uses in the food industry (olive oil and table olives) and its omnipresence in many traditional agrosystems have made this species an economic pillar and cornerstone of Mediterranean agriculture. Specifically, table olives are one of the most important fermented vegetable in Europe and especially in the Mediterranean basin, with an average annual worldwide production of 2.7 millon tonnes in the 2013-2018 season (IOC, 2018). The fermentation of fruits is mainly carried by lactic acid bacteria (LAB) and yeasts in an acid and saline environment, consuming sugars and producing diverse volatile compounds and organic acids (mainly lactic acid) with a considerable influence on the flavour, quality and safety of the final products (Arroyo-López et al., 2008; Garrido-Fernández et al., 1997). Then, fruits are packaged in diverse types of containers (glass, tin, bags, etc.) using preservatives, pasteurization, modified atmospheres or other stabilization systems to increase shelf life.

Herbs, spices and other seasoning material are used in many cases as essential condiments in table olive packaging as they help to enhance their flavor. Many of them, such as garlic or pepper, are also previously fermented independently, and used as stuffed material in pitted olives. Numerous studies have indicated high microbial loads in spices and herbs which could pose a problem for food manufacturers (Baumgartner et al., 2009; Beuchat et al., 2013; Vij et al., 2006). These studies showed the presence of Salmonella sp., considered the principal foodborne pathogen associated with dried herbs and spices, Cronobacter species, verotoxigenic Escherichia coli, Staphylococcus sp., spore forming microorganisms (Bacillus and Clostridium genera), and Listeria 
monocytogenes. All of them were isolated from low-moisture foods including spices and dry herbs.

On the other hand, sodium chloride has been reported to suppress the growth of undesirable bacteria and other spoilage microorganisms by decreasing water activity in foods. During olive processing, salt can also give fruits a crunchier texture, since the salt hardens the pectin in vegetables. Nevertheless, there is scarce information on the microbial load provided by salt in olive elaboration, and how it could affect the stability of the final product. Recently, Benítez-Cabello et al. (2016) reported the presence of resistant bacteria in salt through culture-dependent methods. Species belonging to the Bacillus, Staphylococcus and Enterococcus genera, not detected during the fermentation process, were found in the solid marine salt used by the industry for preparation of brines. Also, some halophilic bacteria such as Halolactibacillus halophilus or species from Marinilactibacillus genus have been previously described during table olive processing (Lucena-Padrós et al., 2015; Lucena-Padrós and Ruiz-Barba, 2016; Rodríguez-Gómez et al., 2017).

So far, most of the studies conducted on microbial biodiversity in foods or condiments have been approached through classical and culture-dependent techniques. However, the use of new molecular techniques in food microbiology, such as cultureindependent methods, complement the studies carried out and allow to overcome the limitations of these traditional methods. In this sense, Next Generation Sequencing techniques represent a change in the way that researchers address microbial ecology in foods. Indeed, the application of metataxonomic analysis allows a detailed understanding of microbial diversity. Specifically, in table olives, diverse metataxonomic studies (Arroyo-López et al., 2016; Cocolin et al., 2013; De Angelis et 
al., 2015; Medina et al., 2016, 2018; Rodríguez-Gómez et al., 2017) have been carried out to determine the bacterial and fungal evolution during olive fermentation, but this approach was never applied to the study of olive dressings or salt used to prepare brines in table olive processing.

The goal of this study is to provide a more precise knowledge of the bacterial biodiversity in some of the main ingredients and dressing material used in table olive packaging through metataxonomic approaches. The possible role of these microorganisms in the final product quality is discussed. Data obtained could be used by industry to design new strategies to improve the quality, safety and stability of the final products.

\section{MATERIALS AND METHODS}

\subsection{Types of samples}

Samples were provided from different table olive producer companies from the regions of Seville and Málaga (Spain) during the 2015/2016 season and transported to the laboratory for analysis in the same day. A total of 19 samples divided in three groups were processed. Five samples were obtained from diverse aromatic herbs (thymus, fennel, and oregano), seven samples from fermented dressings material (sliced red pepper, pepper paste, and garlic) and seven from salts (marine and spring salt). All these samples are ready-to-use by industry and they are directly added to olives as ingredient or stuffed material during the packaging step.

\subsection{DNA extraction}

Five grams of every herb sample was homogenized with $50 \mathrm{~mL}$ of sterile saline solution $(0.9 \% \mathrm{NaCl})$ in a Stomacher ${ }^{\circledR}$ homogenizer (Seward Laboratory Systems, Inc. 
Bohemia, NY, USA) for $2 \mathrm{~min}$, and the aqueous phase was centrifuged at 9,000 $x \mathrm{~g}$ for $15 \mathrm{~min}$. For fermented dressing samples, $25 \mathrm{~g}$ were processed in $50 \mathrm{~mL}$ of sterile saline solution in the same conditions described above. Finally, for salt samples, $25 \mathrm{~g}$ were diluted in $100 \mathrm{ml}$ of distilled water and centrifuged as described above. In all cases, the supernatant was withdrawn and pellets were washed twice with sterile saline solution before stored at $-80^{\circ} \mathrm{C}$ until use.

Microbial genomic DNA was isolated and purified from pellets using the PowerFood ${ }^{\circledR}$ Microbial DNA Isolation Kit (MoBio, Carlsbad, Calif) according to the manufacturer instructions, and was stored at $-20{ }^{\circ} \mathrm{C}$ until use. Prior to sequencing, the amount of purified DNA, that ranged from 5.6 to $42.2 \mathrm{ng} / \mu \mathrm{l}$, was measured using a Qubit fluorometer (Thermo Fisher Scientific, Waltham, USA).

\subsection{Sequencing}

DNA samples were sent for sequencing to FISABIO (Valencia, Spain). 16S rDNA gene amplicons were amplified following the 16S rDNA gene Metagenomic Sequencing Library Preparation Illumina protocol (Part \# 15044223 Rev. A). The genespecific sequences used in this protocol target the $16 \mathrm{~S}$ rDNA gene, both V3 and V4 regions (Klindworth et al., 2013). Libraries were sequenced using a 2x300 bp pairedend run (MiSeq Reagent kit v3 (MS-102-3001) on a MiSeq Sequencer, according to manufacturer's instructions (Illumina). Quality assessment was performed by the use of prinseq-lite program (Schmieder and Edwards, 2011) by applying the following parameters: minimum sequence length of $50 \mathrm{bp}$, trim_qual_right of 30, trim_qual_type of mean and trim_qual_window of $20 \mathrm{bp}$. R1 and R2 from Illumina sequencing where joined using FLASH program (Magoč and Salzberg, 2011) by applying default parameters. 
For the metataxonomic data analysis, the *.fna format files were processed using

121 the Quantitative Insights into Microbial Ecology (QIIME) pipeline (version1.9.1.

122 http://qiime.sourceforge.net/) in a server running Ubuntu v16.04. Sequences were first

123 screened for quality control, being retained these with a length between 220-300 bp and

124 mean sequence Phred quality score $>35$. Any sequence not meeting these parameters

125 was excluded from downstream analyses. Then sequences were sorted by barcode into

126 their respective samples. In order to calculate downstream diversity measures (alpha

127 diversity indices), 16S rRNA Operational Taxonomic Units (OTUs) were defined at the

128 level of $\geq 97 \%$ sequence homology. Chimeric sequences were removed using

129 ChimeraSlayer, and SILVA108 was used as reference sequence database (McDonald et

130 al., 2012). OTUs were assigned using uclust (Edgar, 2010) by using the script

131 pick_de_novo_otus.py. This script produced an OTU mapping file, a representative set

132 of sequences, a sequence alignment file, taxonomy assignment file, a filtered sequence

133 alignment, a phylogenetic tree, and a biom-formatted OTU table. The hierarchical

134 clustering based on population profiles of most common and abundant taxa was

135 performed using UPGMA clustering (Unweighted Pair Group Method with Arithmetic

136 mean). Cyanobacteria and mitochondrial OTUs were removed from the resulting OTU

137 table. Taxonomic abundances within each identified Phylum to genus level were

138 visualized using Krona hierarchical data browser (Ondov et al., 2011)

Alpha diversity indexes were calculated through the alpha_diversity.py script using different metrics (Chao, Observed Species, Shannon, Simpson, Margalef, Good's coverage, PD whole tree) after performing a rarefaction analysis. Rarefied OTU tables 
to 11.400 sequences (lowest number of reads obtained) were used to obtain these alpha diversity metrics.

The OTU tables were collapsed at five taxonomic levels (Phylum, Class, Order,

145 Family, and Genus), exported in tab-delimited text format and analyzed using STAMP

146 v2.0.1 (Parks and Beiko, 2010). A non-supervised principal component analysis (PCA)

147 was conducted after grouping the variables in three categories; fermented dressings

148 (Garlic, pepper and paste of pepper), herbs (thymus, oregano and fennel), and salts

149 (marine and spring salt). In order to determine the unique and shared OTUs between

150 groups, a Venn diagram was constructed on the basis to relative abundance of bacteria

151 on the level of class. An ANOVA/Tukey Kramer (post hoc) test was running to

152 elucidate the taxa association in the different grouping variables. The false discovery

153 rate correction [FDR, (Benjamini and Hochberg, 1995)] was finally applied in all cases

154 and significant differences in taxa were only considered below a $p$-value of 0.05 and 155 a $q$-value below 0.3 .

$156 \quad 3 . \quad$ RESULTS

157

\subsection{Phylogenetic analysis}

This study generated a total of 3,478,486 raw sequences for the 19 samples, 159 from which $3,435,826$ sequences with high quality were recovered after the quality 160 control (98.77\%). From those, we obtained 2,660,444 (83.79\%) sequences that could be assigned into OTUs. Finally, after removing chloroplast and mitochondria, a total of $1,011,230$ sequences $(29.43 \%)$ were used for metataxonomic analysis with a mean of 126,403 sequences per sample. 

diversity indexes. The maximum number of OTUs were assigned to the salt samples $(6,925)$, followed by herb group $(6,744)$, and much further the food fermented group with less of the half of OTUs $(3,072)$. This high number of OTUs found in the salt samples was also translated into greater biodiversity, as shown either by Chao1, Simpson, Shannon, or Margalef indexes. Despite the rarefaction curves analysis, around $97 \%$ of Good's coverage values were obtained for all the samples, which indicated a satisfactory coverage of bacterial diversity.

Krona plots show the relative abundance of taxa at class level for the different group of samples (Figure 1). Bacilli was the dominant bacteria class in the fermented dressings with an average proportion of $64.29 \%$ of the sequences, followed by Gammaproteobacteria (22.84\%) (Figure 1 upper panel). The rest of sequences in this group belonged to minority classes. In the case of herbs, the $61.49 \%$ of the total sequences was covered by the Gammaproteobacteria class, followed by Alphaproteobacteria (20.62\%). Bacilli class was only present in the $3.90 \%$ of the sequences in this type of sample, similar to the Actinobacteria class (5.86\%) (Figure 1 middle panel). Referring to the salt samples, Cytophagia and Halobacteria classes predominated with the 30.77 and $21.77 \%$ of the sequences, respectively. Other classes appeared to a lesser extent, such as Alphaproteobacteria (12.50\%), Deltaproteobacteria (7.60\%) or Gammaproteobacteria (8.61\%) (Figure 1, bottom panel). shared among the three groups of samples at class level. Salt group shared the same number of OTUs with both, fermented and herb groups (16 OTUs; 10.6\%). Meanwhile, fermented dressing and herb groups shared the lowest number of OTUs (5 OTUs; 
$3.3 \%$ ). On the other hand, whereas only a single OTU (non-shared by any other group) appeared in fermented and herbs groups, 57 OTUs (37.7\%) showed to be unique in the salt group. Figure $2 \mathrm{~b}$ shows the unique and shared OTUs between samples after selecting those with more than $1 \%$ representation from all OTUs in at least one of the groups. None of the groups showed unique OTUs with more than $1 \%$ representation. Bacilli, Halobacteria, Flavobacteria, Cytophagia, Gammaproteobacteria, Betaproteobacteria, Deltaproteobacteria, and Clostridia classes were shared by all groups. Meanwhile, Alphaproteobacteria, Actinobacteria, and Sphingobacteria classes were shared exclusively between fermented dressing and salt groups. Lastly, no OTUs with high representation were shared between salt and fermented groups or salt and herbs groups. Table S1 (supplementary material) shows the total OTUs assigned at class level, shared and unique among the three groups of samples. Specifically, Cytophagya, Bacilli, Gammaproteobacteria, Deltaproteobacteria, Sphingobacteria, and Halobacteria classes were found to be over-represented in these communities (Figure 3 and Figure S1 in supplementary material) being the only classes with high representation that showed significant differences among the three groups. Box-andwhisker plot showing the difference in mean proportions among the different groups is shown in Figure 4. Significant differences were found between salt samples compared to the other groups. This was due to a higher presence of Cytophagia, Sphingobacteria, Deltaproteobacteria, and Halobacteria classes. No significant differences in the presence of these classes were found between fermented dressing and herb groups. However, the presence of Bacilli class in fermented group was highly significant in this group respecting to salt and herbs group. The difference in the proportion of sequences of the class of Bacilli between salt and herbs was not statistically significant. Finally, the proportion of Gammaproteobacteria was statistically higher in herbs group 
respecting fermented and salt groups. No significant differences in the proportion of

214 Gammaproteobacteria were found between fermented and salt groups. one of the samples. Pseudomonas genus and Enterobacteriaceae family predominated in most samples of herbs. The first one showed $40.04 \%$ of sequences in thyme, $22.29 \%$ in oregano, and $20.58 \%$ in fennel. The non-assigned genus was majority in the fennel samples with $50.62 \%$ of the sequences. In thyme and oregano the representation of this genus was 13.44 and $19.35 \%$ of sequences, respectively. Pseudomonas was also found in fermented dressing samples in less proportion. However the genus of Enterobacteriaceae family appeared in a relative high proportion in pepper paste $(12.49 \%)$ and garlic (9.52\%). Sphingomonas genus was also found exclusively in thyme (8.91\%) and oregano (14.77\%) samples. The presence of $5.55 \%$ of Vibrio genre sequences in pepper is noteworthy. Lactobacillus, Weisella and Pediococcus were the predominant genera in the fermented dressing samples, except in the sample of pepper paste, in which Pediococcus genus was only found in $0.38 \%$ of sequences. The proportion of sequences of Lactobacillus, Weisella and Pediococcus genera in pepper was $27.06,25.89$ and $21.33 \%$ respectively, and $22.88,26.27$ and $18.86 \%$ in garlic. The lack of presence of Pediococcus sp. in pepper paste was complemented by other OTUs with less of $1 \%$ of the total sequences and a higher proportion of the non-assigned genus of Enterobacteriaceae. A higher biodiversity of OTUs with less of 1\% of representation was found in salt samples. Salinibacter was the most representative genus, with 54.87 and $34.49 \%$ of sequences in spring and marine salt samples, respectively. 

the diverse samples, suggests a right selection of data for each group. PCA generated mainly two components. The first and second principal components (PC1 and PC2) explained $80 \%$ of the total variability of the data set. PC1, which explained $45.3 \%$ of

241 the variance had the highest positive loading of herbs samples, and PC2 $(34.7 \%$ of the

242 variance), had the highest positive loading of fermented dressing and salt sample. Similar positive loading of fermented dressing samples was shared by the two components. A clear cluster of communities of fermented, herb and salt samples was observed. However, two of the herbs group samples (Oregano, Thymus) were found to be closer to salt samples.

\section{DISCUSSION}

New generation sequencing techniques, based on independent culture techniques, have meant a revolution for the knowledge of the microbiota present in foods. Until now, the classical methodology only allowed us to know the presence of some cultivable microbial groups, what has kept us blind to the real biodiversity understanding of a given sample. The current challenge is not only to determine the presence or absence of the microorganisms in a sample, but to clarify the role that they could develop in relation to quality and safety aspects. The initial data reveal the great difference in the bacterial population observed among the three types of samples analyzed (fermented dressings, aromatic herbs, and salts). We must bear in mind that the methodology used allows us to determine the presence of DNA of the species in the samples, but this is not a guarantee of the metabolic activity of the microorganisms. With the NGS procedure analyzed, we do not have the certainty that the microorganisms are alive or dead. However, high proportions of DNA sequences 
suggest that at some point microorganisms have been present in high concentrations, indicating a possible important role in terms of activity that may represent a risk for the product in the case of the presence of genera with pathogenic species or strains, also due to the presence of possible toxins produced by them.

Pseudomonas genus and Enterobacteriaceae family represent the two major groups of saprophytic bacteria found on the surface of freshly harvested plants (Blackburn et al., 2006). The high presence of both groups of microorganisms in the aromatic herbs analyzed in this work is not surprising. Pseudomonas genus is considered as ubiquitous due to its metabolic diversity and genome plasticity (Madigan et al., 1997). In general, species belonging to this genus are innocuous to humans but there are also opportunistic pathogens for humans and plants, such as P. aeruginosa and P. syringae. Several species of this group can cause food spoilage. (Raposo et al., 2016). The presence of Pseudomonas genus during fermentation of Aloreña de Málaga table olives has been previously described (Abriouel et al., 2011; Medina et al., 2016). However, no information about the origin was given. Our data could explain the presence of this genus in table olives, since these condiments are common ingredients in the elaboration of Aloreña de Málaga packaging. On the other hand, the Enterobacteriaceae family, although its presence does not necessarily imply a risk, it contains a large number of pathogenic species, such as E. coli, Salmonella sp. or Erwinia sp., among others. The abundance of Enterobacteriaceae sequences in the group of herbs was high, especially in fennel, which has been previously reported as a carrier of these microorganisms (Sospedra et al., 2010).

Weissella, Pediococcus and Lactobacillus are some of the typical genera commonly found in fermented foods (Erten et al., 2017). Our results support the 
presence of these microorganisms as the most representative genera in samples of fermented dressing. All of them belong to the group of bacteria generally known as lactic acid bacteria. Apart from a perceived technical role of certain Weissella species involved in such traditional fermentations, specific strains are also receiving attention as potential probiotics (Fusco et al., 2015). Weisella genus has been reported commonly in fermented food, such as W. koreensis, the dominant specie in the fermentation of Kimchi, a fermented food made with red pepper powder and garlic, among other ingredients (Kang et al., 2015). These researchers also reported that red pepper powder had a strong influence in the abundance of these species. On the other hand, while some Lactobacillus species have been described as spoilage microorganisms in some fermented food (Andreevskaya et al., 2018; Gram et al., 2002). It is known that most species of Lactobacillus and Pediococcus play an important role in the processes of conservation and fermentation of food by lowering the $\mathrm{pH}$ and producing bacteriocins, which may prevent the growth of pathogenic and spoilage microorganisms (Fontana et al., 2005; Papagianni and Anastasiadou, 2009; Sivakumar et al., 2010)

Vibrio sp. was found in a high abundance in one of the samples of pepper. Several species of this genus have been described as pathogenic, causing diseases of the digestive tract (Jones et al., 2017). Recently, Benítez-Cabello et al. (2016) reported the presence of Vibrio vulnificus and Vibrio sp., through RT-PCR-DGGE technique in both, brines and fruits during table olive processing. Isolates from Vibrio

306 furnissii and Vibrio fluvialis were also found by Lucena-Padrós et al. (2014) during 307 the initial stage of the table olive fermentation; they were reported as human pathogens, causing acute gastroenteritis (Ballal et al., 2017; Ramamurthy et al., 2014). 

bacterial diversity in this group of samples. The genus Salinibacter was the most representative in salt samples and was described for the first time by Antón et al.

312 (2002), being S. ruber the only specie of this genus. Recently, Medina et al. (2016)

313 described the presence of the genus during the Aloreña de Málaga table olive 314 processing in brine and fruit samples. Lucena-Padrós and Ruiz-Barba (2016) studied the 315 diversity of halophilic bacteria in Spanish-style green table-olive fermentations, 316 revealing the presence of Amphibacillus, Natronobacillus,

317 Catenococcus and Streptohalobacillus genera among others, but, no evidence of the 318 presence of Salinibacter was reported. This work elucidated that the salt used for 319 preparing the cover brines could be the possible origin of Salinibacter in table olives.

\section{Conclusions}

322 load directly into the packaging of the olive, including spoilage microorganisms that 323 may alter the stability of the table olive packaging.

\section{Acknowledgements} 2013-48300-R: www.olifilm.science.com.es) AB-C and JB-G thanks the Spanish

327 Ministry of Economy and Competitiveness for their FPI grant and JDC-Incorp. contract, respectively. BC-D thanks the Andalusian Ministry of Economy, Science and Innovation by her pre-doctoral contract.

\section{References}


Abriouel, H., Benomar, N., Lucas, R., \& Gálvez, A. (2011). Culture-independent study of the diversity of microbial populations in brines during fermentation of naturallyfermented Aloreña green table olives. International Journal of Food Microbiology, 144(3), 487-496. https://doi.org/10.1016/j.ijfoodmicro.2010.11.006

Andreevskaya, M., Jääskeläinen, E., Johansson, P., Ylinen, A., Paulin, L., Björkroth, J., \& Auvinen, P. (2018). Food spoilage-associated Leuconostoc, Lactococcus, and Lactobacillus species display different survival strategies in response to competition. Applied and Environmental Microbiology,84(13), e00554-18. https://doi.org/10.1128/AEM.00554-18

Antón, J., Oren, A., Benlloch, S., Rodríguez-Valera, F., Amann, R., \& Rosselló-Mora, R. (2002). Salinibacter ruber gen. nov., sp. nov., a novel, extremely halophilic member of the Bacteria from saltern crystallizer ponds. International Journal of Systematic and Evolutionary Microbiology, 52(2), 485-491. https://doi.org/10.1099/00207713-52-2-485

Arroyo-López, F. N., Querol, A., Bautista-Gallego, J., \& Garrido-Fernández, A. (2008). Role of yeasts in table olive production. International journal of food microbiology, 128(2), 189-196. https://doi.org/10.1016/j.ijfoodmicro.2008.08.018

Arroyo-López, F. N., Medina, E., Ruiz-Bellido, M. Á., Romero-Gil, V., MontesBorrego, M., \& Landa, B. B. (2016). Enhancement of the knowledge on fungal communities in directly brined Aloreña de Málaga green olive fermentations by metabarcoding analysis. PloS one, 11(9), e0163135. https://doi.org/10.1371/journal.pone.0163135

Ballal, M., Shetty, V., Bangera, S. R., Prabhu, M., \& Umakanth, S. (2017). Vibrio furnissii, an emerging pathogen causing acute gastroenteritis: a Case Report. JMM 
Baumgartner, A., Grand, M., Liniger, M., \& Iversen, C. (2009). Detection and frequency of Cronobacter spp.(Enterobacter sakazakii) in different categories of ready-to-eat foods other than infant formula. International journal of food microbiology, 136(2), 189-192. https://doi.org/10.1016/j.ijfoodmicro.2009.04.009

Benítez-Cabello, A., Bautista-Gallego, J., Garrido-Fernández, A., Rantsiou, K., Cocolin, L., Jiménez-Díaz, R., \& Arroyo-López, F. N. (2016). RT-PCR-DGGE analysis to elucidate the dominant bacterial species of industrial Spanish-style green table olive fermentations. Frontiers in microbiology, 7, 1291. https://doi.org/10.3389/fmicb.2016.01291

Benjamini, Y., Hochberg, Y., 1995. Controlling the false discovery rate: a practical and powerful approach to multiple testing. J. R. Stat. Soc. Ser. B 289-300.

Beuchat, L. R., Komitopoulou, E., Beckers, H., Betts, R. P., Bourdichon, F., Fanning, S., ... \& Ter Kuile, B. H. (2013). Low-water activity foods: increased concern as vehicles of foodborne pathogens. Journal of food protection, 76(1), 150-172. https://doi.org/10.4315/0362-028X.JFP-12-211

Cocolin, L., Alessandria, V., Botta, C., Gorra, R., De Filippis, F., Ercolini, D., \& Rantsiou, K. (2013). NaOH-debittering induces changes in bacterial ecology during table olives fermentation. PLoS One, 8(7), e69074.

De Angelis, M., Campanella, D., Cosmai, L., Summo, C., Rizzello, C.G., Caponio, F., 2015. Microbiota and metabolome of un-started and started Greek-type fermentation of Bella di Cerignola table olives. Food Microbiol. 52, 18-30. https://doi.org/10.1371/journal.pone.0069074 
378 De W Blackburn, C. (Ed.). (2006). Food spoilage microorganisms. Woodhead $379 \quad$ Publishing.

380 Edgar, R. C. (2010). Search and clustering orders of magnitude faster than https://doi.org/10.1093/bioinformatics/btq461

Erten, H., Agirman, B., Boyaci-Gündüz, C. P., \& Ghorbal, A. B. (2017). Regional fermented vegetables and fruits in Europe. Lactic Acid Fermentation of Fruits and Vegetables, 205-235.

Fontana, C., Cocconcelli, P. S., \& Vignolo, G. (2005). Monitoring the bacterial population dynamics during fermentation of artisanal Argentinean sausages. International journal of food microbiology,103(2), 131-142. https://doi.org/10.1016/j.ijfoodmicro.2004.11.046

Fusco, V., Quero, G. M., Cho, G. S., Kabisch, J., Meske, D., Neve, H., ... \& Franz, C. M. (2015). The genus Weissella: taxonomy, ecology and biotechnological potential. Frontiers in microbiology, 6, 155. https://doi.org/10.3389/fmicb.2015.00155

Garrido-Fernández, A., Adams, M.R., Fernández-Díez, M.J., 1997. Table olives: production and processing. Springer Science \& Business Media.

Gram, L., Ravn, L., Rasch, M., Bruhn, J. B., Christensen, A. B., \& Givskov, M. (2002). Food spoilage_-interactions between food spoilage bacteria. International journal of food microbiology, 78(1-2), 79-97. https://doi.org/10.1016/S01681605(02)00233-7

International Olive Oil Council (IOC) Updates Series of World Statistics on Production, Imports, Exports and Consumption. [(accessed on 23 October 

http://www.internationaloliveoil.org/estaticos/view/132-world-table-olive-figures

Jones, J. L. (2017). In Dodd C. E. R., Aldsworth T., Stein R. A., Cliver D. O. and Riemann $\quad$ H. $\quad$ P.(Eds.), Chapter 11 - vibrio Academic Press. doi: https://doi.org/10.1016/B978-0-12-385007-2.00011-5

Kang, B. K., Cho, M. S., Ahn, T. Y., Lee, E. S., \& Park, D. S. (2015). The influence of red pepper powder on the density of Weissella koreensis during kimchi fermentation. Scientific reports, 5, 15445.

Klindworth, A., Pruesse, E., Schweer, T., Peplies, J., Quast, C., Horn, M., \& Glöckner, F. O. (2013). Evaluation of general 16S ribosomal RNA gene PCR primers for classical and next-generation sequencing-based diversity studies. Nucleic acids research, 41(1), e1-e1. https://doi.org/10.1093/nar/gks808

Lucena-Padrós, H., Caballero-Guerrero, B., Maldonado-Barragán, A., \& Ruiz-Barba, J. L. (2014). Microbial diversity and dynamics of Spanish-style green table-olive fermentations in large manufacturing companies through culture-dependent techniques. Food microbiology, 42, 154-165. https://doi.org/10.1016/j.fm.2014.03.020

Lucena-Padrós, H., Jiménez, E., Maldonado-Barragán, A., Rodríguez, J. M., \& RuizBarba, J. L. (2015). PCR-DGGE assessment of the bacterial diversity in Spanishstyle green table-olive fermentations. International journal of food microbiology, 205, 47-53. https://doi.org/10.1016/j.ijfoodmicro.2015.03.033

Lucena-Padrós, H., \& Ruiz-Barba, J. L. (2016). Diversity and enumeration of halophilic and alkaliphilic bacteria in Spanish-style green table-olive fermentations. Food microbiology, 53, 53-62. https://doi.org/10.1016/j.fm.2015.09.006 
Madigan, M.T., Martinko, J.M., Parker, J., 1997. Brock biology of microorganisms. Prentice hall Upper Saddle River, NJ.

Magoč, T., \& Salzberg, S. L. (2011). FLASH: fast length adjustment of short reads to improve genome assemblies. Bioinformatics, 27(21), 2957-2963. https://doi.org/10.1093/bioinformatics/btr507

McDonald, D., Price, M. N., Goodrich, J., Nawrocki, E. P., DeSantis, T. Z., Probst, A., ... \& Hugenholtz, P. (2012). An improved Greengenes taxonomy with explicit ranks for ecological and evolutionary analyses of bacteria and archaea. The ISME journal, 6(3), 610.

Medina, E., Ruiz-Bellido, M. A., Romero-Gil, V., Rodríguez-Gómez, F., MontesBorrego, M., Landa, B. B., \& Arroyo-López, F. N. (2016). Assessment of the bacterial community in directly brined Aloreña de Málaga table olive fermentations by metagenetic analysis. International journal of food microbiology, 236, 47-55. https://doi.org/10.1016/j.ijfoodmicro.2016.07.014

Medina, E., Brenes, M., García-García, P., Romero, C., \& de Castro, A. (2018). Microbial ecology along the processing of Spanish olives darkened by oxidation. Food Control, 86, 35-41. https://doi.org/10.1016/j.foodcont.2017.10.035

Ondov, B. D., Bergman, N. H., \& Phillippy, A. M. (2011). Interactive metagenomic visualization in a Web browser. BMC bioinformatics, 12(1), 385.

Papagianni, M., \& Anastasiadou, S. (2009). Pediocins: The bacteriocins of Pediococci. Sources, production, properties and applications. Microbial cell factories, 8(1), 3. https://doi.org/10.1186/1475-2859-8-3

Parks, D. H., \& Beiko, R. G. (2010). Identifying biologically relevant differences between metagenomic communities. Bioinformatics, 26(6), $715-721$. 
451 Ramamurthy, T., Chowdhury, G., Pazhani, G. P., \& Shinoda, S. (2014). Vibrio fluvialis: 452 an emerging human pathogen. Frontiers in microbiology, 5, 91. https://doi.org/10.3389/fmicb.2014.00091

454

455

Raposo, A., Pérez, E., de Faria, C. T., Ferrús, M. A., \& Carrascosa, C. (2017). Food Spoilage by Pseudomonas spp.-An Overview. Food Borne Pathogens and Antibiotic Resistance.

Rodríguez-Gómez, F., Ruiz-Bellido, M. Á., Romero-Gil, V., Benítez-Cabello, A., Garrido-Fernández, A., \& Arroyo-López, F. N. (2017). Microbiological and Physicochemical Changes in Natural Green Heat-Shocked Aloreña de Málaga Table Olives. Frontiers in microbiology, 8 , 2209. https://doi.org/10.3389/fmicb.2017.02209

Schmieder, R., \& Edwards, R. (2011). Quality control and preprocessing of metagenomic datasets. Bioinformatics, 27(6), 863-864. https://doi.org/10.1093/bioinformatics/btr026

Sivakumar, N., \& Saif, A. B. (2010). Partial characterization of bacteriocins produced by Lactobacillus acidophilus and Pediococcus acidilactici. Brazilian Archives of Biology and Technology, 53(5), 1177-1184. http://dx.doi.org/10.1590/S151689132010000500023

Sospedra, I., Soriano, J. M., \& Mañes, J. (2010). Assessment of the microbiological safety of dried spices and herbs commercialized in Spain. Plant foods for human nutrition, 65(4), 364-368.

Vij, V., Ailes, E., Wolyniak, C., Angulo, F. J., \& Klontz, K. C. (2006). Recalls of spices due to bacterial contamination monitored by the US Food and Drug 
Administration: the predominance of Salmonellae. Journal of food protection, 69(1), 233-237. https://doi.org/10.2989/20702620.2010.547268

\section{Figure legends}

Figure 1. Krona plots showing the relative abundance of taxa (\%) at class level for fermented dressing (upper panel), herbs (middle panel) and salts (bottom panel) groups.

Figure 2. Venn diagrams showing the total number of unique and shared bacterial OTUs at class level (a) and unique and shared bacterial class with percentage $>1$ representation in at least one of the groups (b), between fermented dressing samples (F), salt (S), and herb (H) groups.

Figure 3. ANOVA Tukey-Kramer post hoc comparison test executed with STAMP v2.0.1 to elucidate significant differences for Cytophagia class in the diverse group of samples. Significant differences were only considered for $\mathrm{p} \leq 0.05$ and a $\mathrm{q}$-value below 0.3. Meaning of symbols: Cross, star and horizontal line inside box denoted for outlier, mean, and median of the percentage of sequences obtained. F, fermented dressing group; $\mathrm{H}$, herbs group; $\mathrm{S}$, salt group of seasoning.

Figure 4. Comparison of bacterial community profiles at the levels of class between OTUs (defined at $>97 \%$ ) which showed significant differences and only present in appreciable numbers within communities sampled from seasoning groups. Analysis was performed using STAMP and significant differences in taxa were only considered below a $p$-value of 0.05 and a $q$-value below 0.3. Percentage of mean proportion of taxa by groups (left) and difference in the mean proportion (right) are illustrated. 
500 Figure 5. Relative abundance of sequences (\%) to genus or family levels obtained by 501 metataxonomic analysis from thymus $(\mathrm{T})$, oregano $(\mathrm{O})$, fennel $(\mathrm{Fn})$, pepper $(\mathrm{P})$, 502 pasta pepper (PP), garlic (G), marine salt (SM) and salt spring (SS) samples.

503 Figure 6. PCA plot comparing class-level taxonomic profiles of the different group of 504 samples ( $\mathrm{F}$, fermented dressing group; $\mathrm{H}$, herbs group; $\mathrm{S}$, salt group). 
Table 1. Number of assigned sequences and OTUs at class level (after chloroplast and mitochondria removal), alpha diversity indices and estimated sample coverage for $16 \mathrm{~S}$ amplicons (bacteria) according to the group of samples ${ }^{1}$ and single samples ${ }^{2}$

\begin{tabular}{cccccccccc}
\hline $\begin{array}{c}\text { Sample } \\
\text { reference }\end{array}$ & chao1 & PD_whole_tree & shannon & simpson & margalef & dominance & Counts/sample & observed_otus & goods_coverage \\
\hline $\mathbf{S}(n=7)$ & 3892.07 & 109.55 & 6.32 & 0.92 & 137.92 & 0.08 & 150034.21 & 6925.42 \\
$\mathbf{F}(n=7)$ & 3444.37 & 51.63 & 4.40 & 0.80 & 81.29 & 0.20 & 71647.11 & 3072.89 \\
$\mathbf{H}(\mathrm{n}=5)$ & 3695.20 & 54.79 & 5.45 & 0.89 & 110.05 & 0.11 & 142579.17 & 6744.83 & 0.97 \\
\hline
\end{tabular}

${ }^{1}$ S-Salt, F-Fermented dressing, H-Herbs.

\begin{tabular}{|c|c|c|c|c|c|c|c|c|c|}
\hline $\begin{array}{l}\text { Type of } \\
\text { sample }\end{array}$ & chao1 & PD_whole_tree & shannon & simpson & margalef & dominance & Counts/sample & observed_otus & goods_coverage \\
\hline$S M(n=4)$ & 4920.65 & 116.21 & 7.31 & 0.97 & 166.54 & 0.03 & 150772.75 & 8818.50 & 0.96 \\
\hline SS $(n=3)$ & 2863.49 & 102.89 & 5.34 & 0.88 & 109.30 & 0.12 & 149295.67 & 5032.33 & 0.98 \\
\hline $\mathbf{G}(\mathrm{n}=3)$ & 2877.99 & 50.40 & 4.76 & 0.83 & 79.29 & 0.17 & 54753.33 & 2427.67 & 0.97 \\
\hline$P(n=2)$ & 3725.88 & 56.81 & 3.74 & 0.74 & 87.57 & 0.26 & 96103.50 & 4160.00 & 0.97 \\
\hline$P P(n=2)$ & 3729.25 & 47.69 & 4.70 & 0.83 & 77.02 & 0.17 & 64084.50 & 2631.00 & 0.97 \\
\hline$O(n=2)$ & 2907.21 & 68.01 & 6.01 & 0.94 & 115.61 & 0.06 & 94946.50 & 4083.50 & 0.97 \\
\hline$T(n=2)$ & 4703.38 & 56.30 & 5.74 & 0.90 & 118.83 & 0.10 & 212358.00 & 10462.00 & 0.96 \\
\hline Fn $(n=1)$ & 3475.01 & 40.05 & 4.62 & 0.82 & 95.70 & 0.18 & 120433.00 & 5689.00 & 0.96 \\
\hline
\end{tabular}

${ }^{2}$ SM-marine salt, SS-spring salt, G-garlic, P-pepper, PP-pepper paste, O-oregano, T-thymus, Fn-Fennel.

${ }^{\mathrm{a}}$ Alpha diversity metrics were obtained after rarefaction of the samples to 11400 sequences. 
Figure 1
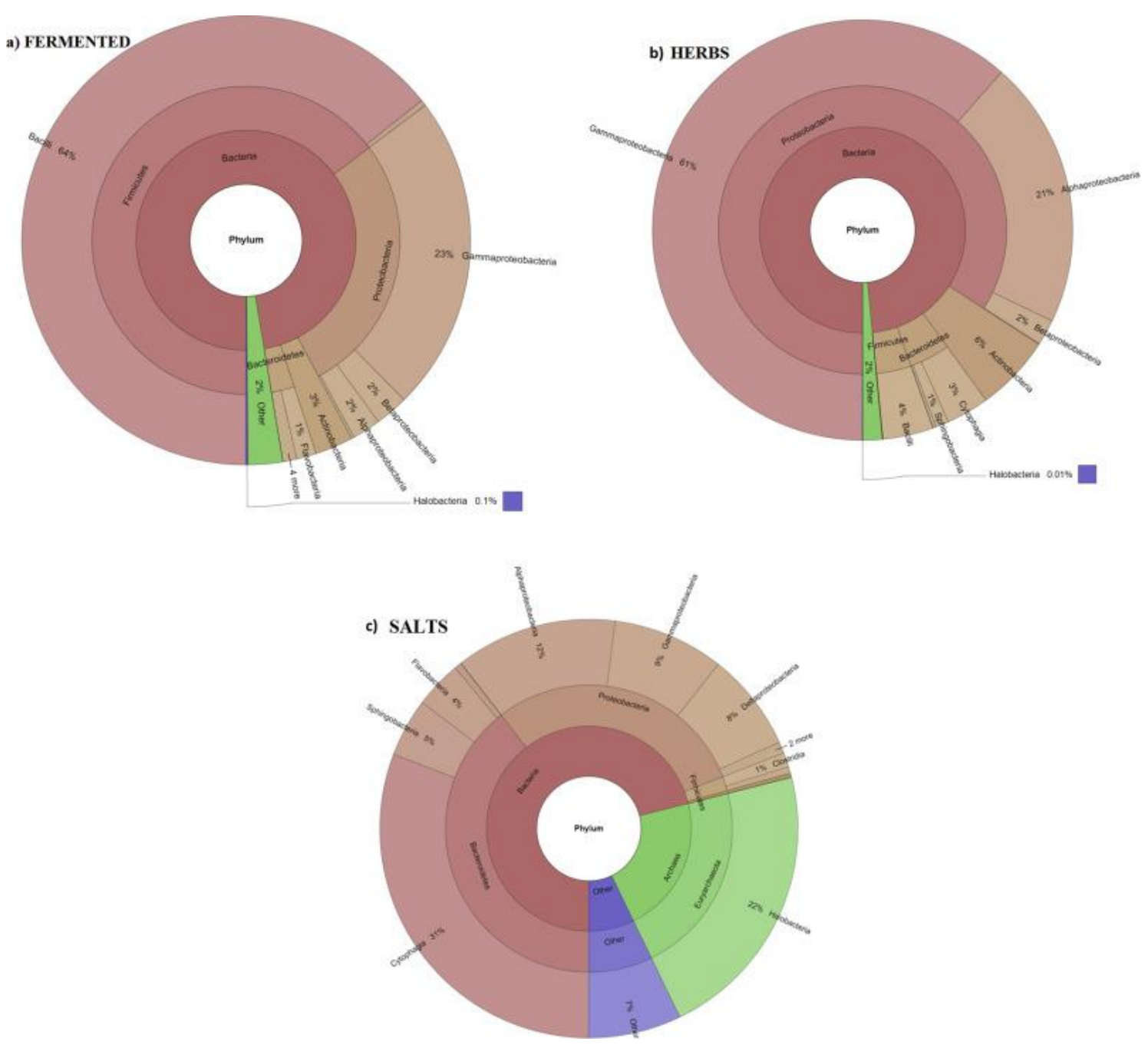
Figure 2

a

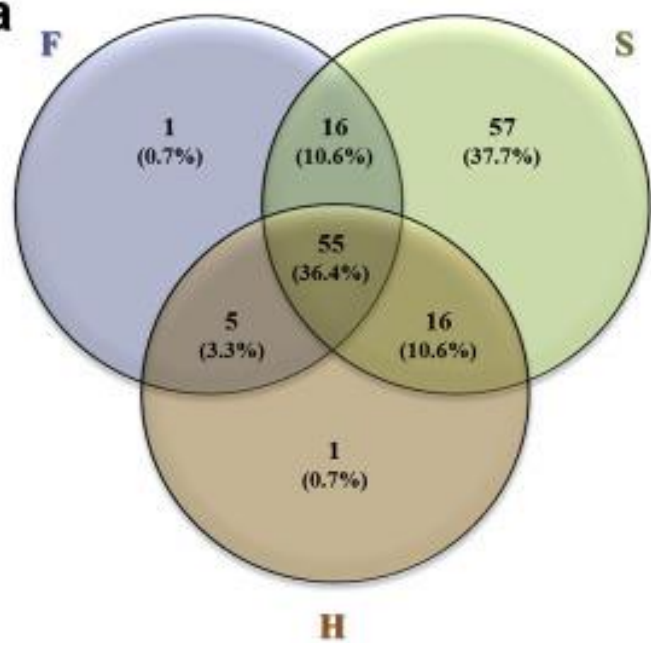

b

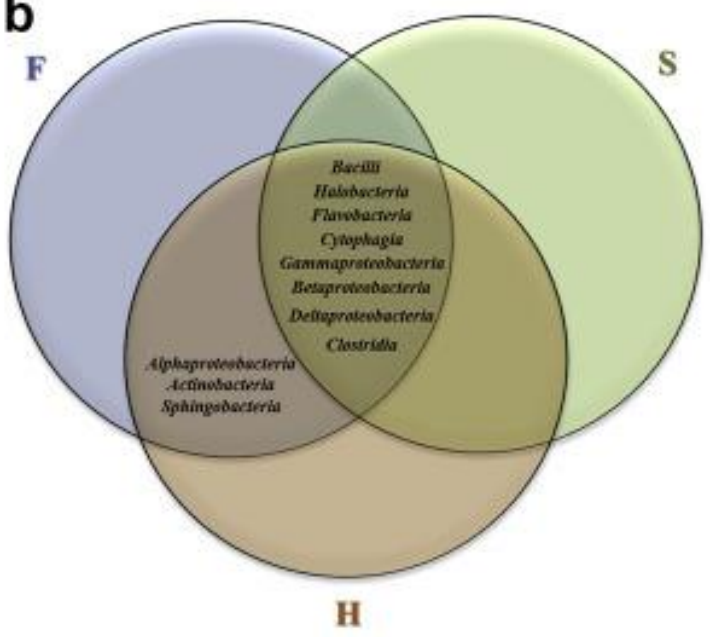


Figure 3

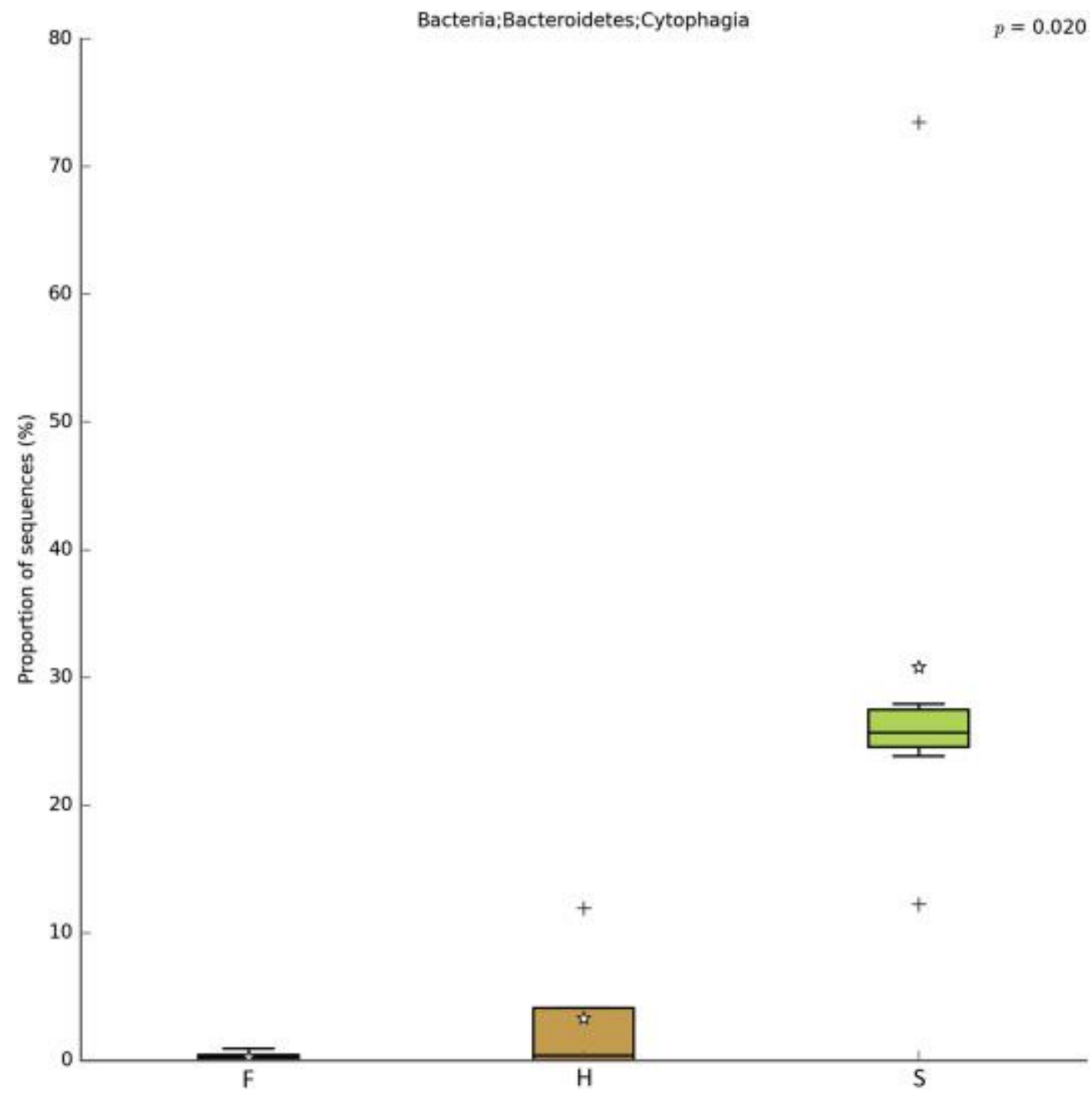


Figure 4

Bacteria;Proteobacteria;Deltaproteobacteria $\quad 95 \%$ confidence intervals

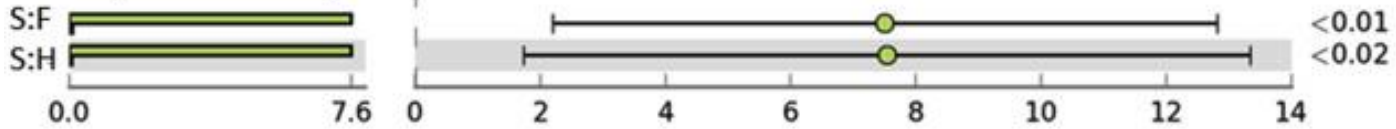

Archaea;Euryarchaeota; Halobacteria

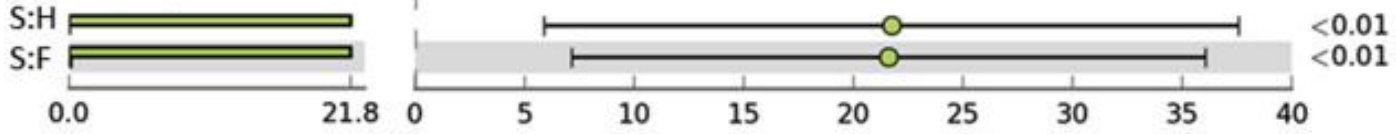

Bacteria;Bacteroidetes;Cytophagia
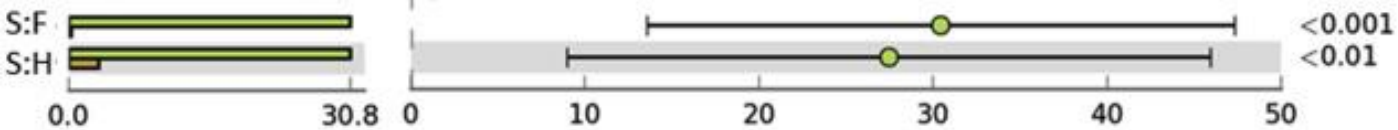

Bacteria;Firmicutes;Bacilli

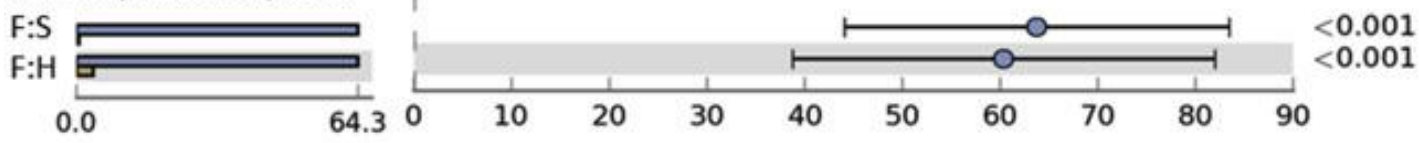

Bacteria;Proteobacteria;Gammaproteobacteria

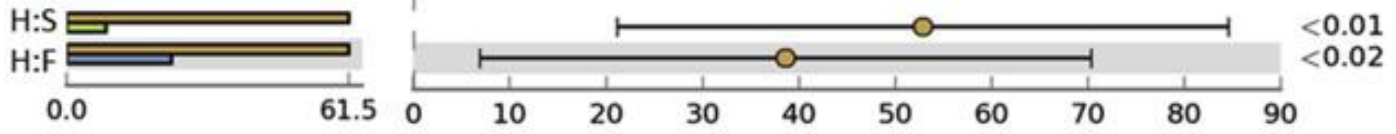

Bacteria;Bacteroidetes;Sphingobacteria

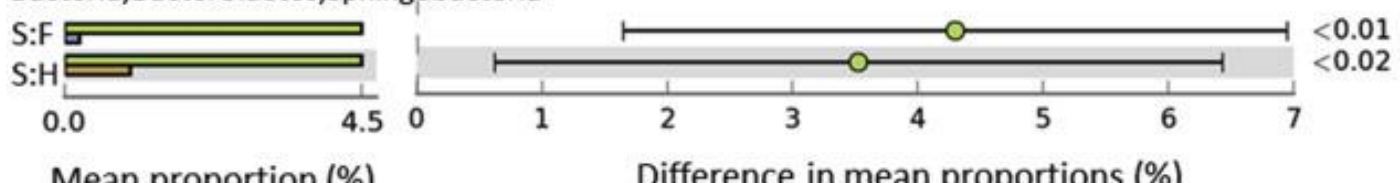

Mean proportion (\%) Difference in mean proportions (\%) 
Figure 5

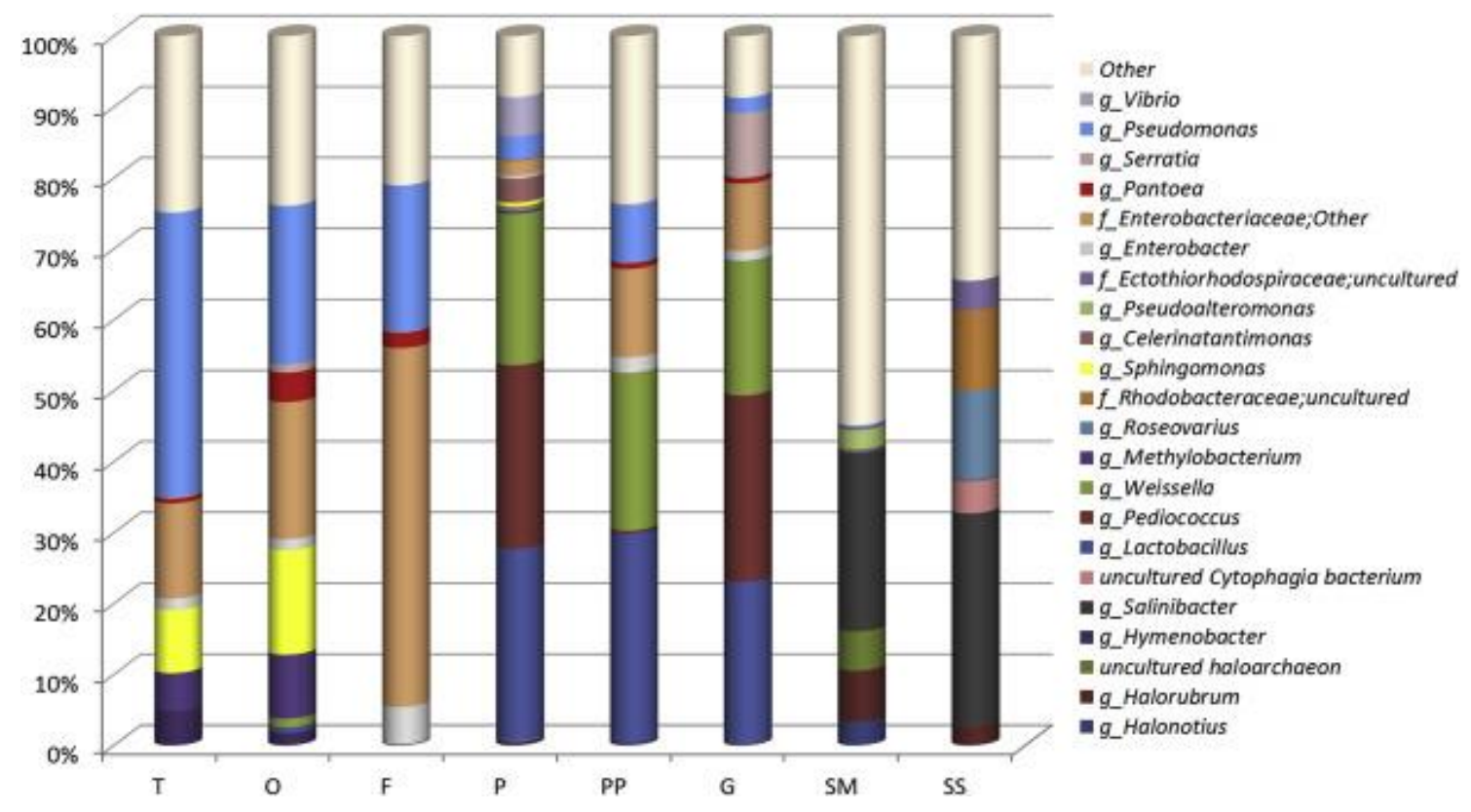


Figure 6

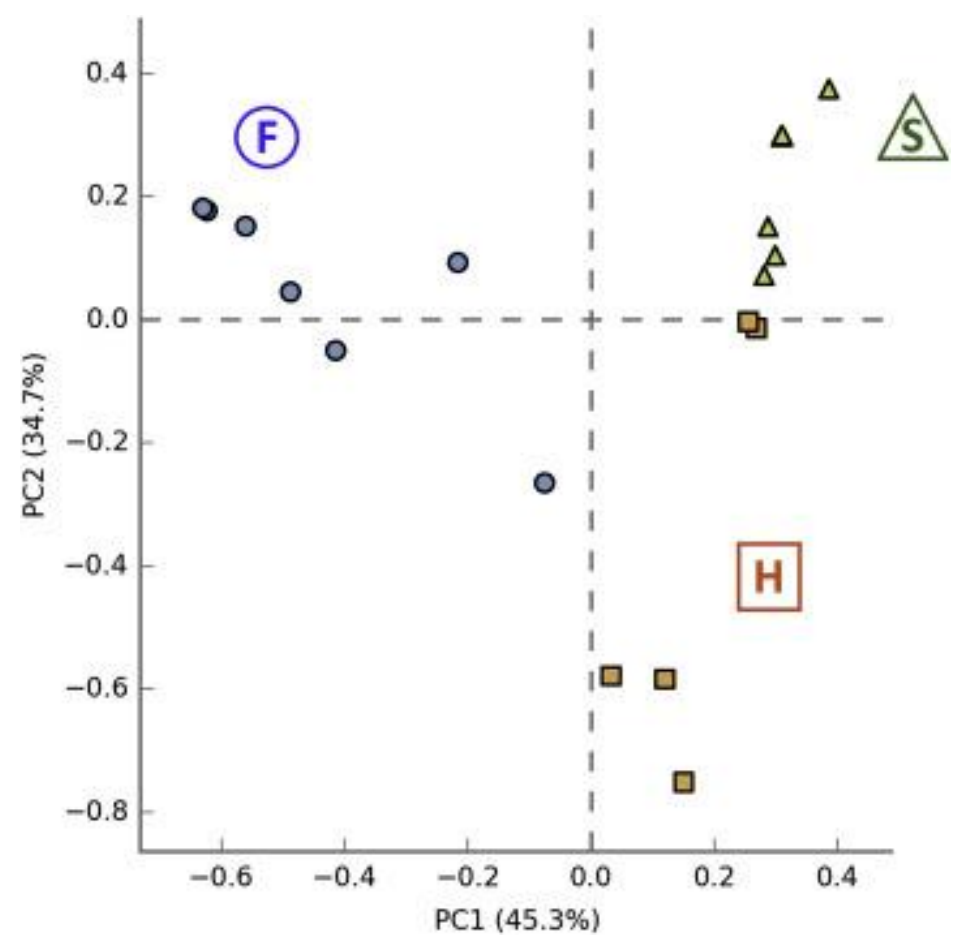

